\title{
Ionic conductivity of nanocrystalline yttria-stabilized zirconia: Grain boundary and size effects
}

\author{
O. J. Durá, ${ }^{1}$ M. A. López de la Torre, ${ }^{1}$ L. Vázquez, ${ }^{2}$ J. Chaboy, ${ }^{3}$ R. Boada,${ }^{3}$ A. Rivera-Calzada, ${ }^{4}$ J. Santamaria, ${ }^{4}$ \\ and C. Leon ${ }^{4}$ \\ ${ }^{1}$ Departamento de Física Aplicada and INEI, Universidad de Castilla-la Mancha, 13071 Ciudad Real, Spain \\ ${ }^{2}$ Instituto de Ciencia de Materiales de Madrid, CSIC, Cantoblanco, 28049 Madrid, Spain \\ ${ }^{3}$ Instituto de Ciencia de Materiales de Aragón, Consejo Superior de Investigaciones Científicas, CSIC-Universidad de Zaragoza, \\ 50009 Zaragoza, Spain \\ ${ }^{4}$ GFMC, Departamento de Física Aplicada III, Facultad de Física, Universidad Complutense de Madrid, Campus Moncloa, \\ 28040 Madrid, Spain
}

(Received 17 November 2009; revised manuscript received 17 April 2010; published 10 May 2010)

\begin{abstract}
We report on the effect of grain size on the ionic conductivity of yttria-stabilized zirconia samples synthesized by ball milling. Complex impedance measurements, as a function of temperature and frequency are performed on $10 \mathrm{~mol} \%$ yttria-stabilized zirconia nanocrystalline samples with grain sizes ranging from 900 to $17 \mathrm{~nm}$. Bulk ionic conductivity decreases dramatically for grain sizes below $100 \mathrm{~nm}$, although its activation energy is essentially independent of grain size. The results are interpreted in terms of a space-charge layer resulting from segregation of mobile oxygen vacancies to the grain-boundary core. The thickness of this space-charge layer formed at the grain boundaries is on the order of $1 \mathrm{~nm}$ for large micron-sized grains but extends up to $7 \mathrm{~nm}$ when decreasing the grain size down to $17 \mathrm{~nm}$. This gives rise to oxygen vacancies depletion over a large volume fraction of the grain and consequently to a significant decrease in oxide-ion conductivity.
\end{abstract}

DOI: 10.1103/PhysRevB.81.184301

PACS number(s): $66.30 . \mathrm{H}-$

\section{INTRODUCTION}

Reducing the operation temperature of solid oxide fuel cells (SOFCs) is nowadays a major goal of technological importance for their widespread use in power generation. This has triggered an intense research effort aimed to reach a better understanding of the dynamics of mobile oxygen ions in solids, together with the search of novel or improved materials with higher oxide-ion conductivity which allows them to work efficiently as electrolytes near room temperature. ${ }^{1-4}$ Among oxide-ion conductors, those of anion-deficient fluorite structure such as yttria-stabilized zirconia [YSZ: $\left(\mathrm{Y}_{2} \mathrm{O}_{3}\right)_{x}\left(\mathrm{ZrO}_{2}\right)_{1-x}$ ] are extensively used today as electrolytes in SOFCs. ${ }^{1,2}$ These materials are characterized by a large number of mobile oxygen vacancies, which are randomly distributed in the structure and thus give rise to a completely disordered anion (oxygen) sublattice. Doping with $\mathrm{Y}_{2} \mathrm{O}_{3}$ is known to stabilize the cubic fluorite structure of $\mathrm{ZrO}_{2}$ and to supply the oxygen vacancies responsible for the ionic conduction. ${ }^{5}$ Long-range migration of oxygen ions takes place by thermally activated hopping to adjacent oxygen vacancies, what yields a dc conductivity obeying an Arrhenius law $\sigma_{\mathrm{dc}}=\sigma_{\infty} \exp \left(-E_{\mathrm{dc}} / k T\right)$. Thus, increasing conductivity at a given temperature would require increasing the prefactor $\sigma_{\infty}$ and/or decreasing the activation energy $E_{\mathrm{dc}}$. The usual approach to increase the conductivity of the electrolyte is to chemically substitute elements that preferentially increase the concentration of the mobile specie. ${ }^{1}$ This has recently allowed reduction in the operating temperature to the range of $500-750{ }^{\circ} \mathrm{C}$ by using gadolinium doped ceria and other ionically conducting materials ${ }^{6-11}$ but this temperature is still too high for many technological applications. Unfortunately, there is often an optimum level of doping beyond which the conductivity begins to decrease.
Nanoionics has emerged as a promising path toward enhanced values of the ionic conductivity resulting from confinement in nanostructured materials ${ }^{12-14}$ and which is expected to have a large impact on the next generation of electrochemical devices such as lithium batteries ${ }^{15}$ or fuel cells. ${ }^{16-18}$ Space charge effects are an interesting family of nanoionic size effects which show up when sample dimensions are comparable to the extension of the space-charge region. ${ }^{12,19,20}$ It is well known that the energy for defect generation may be different at surfaces or boundaries. In ionic compounds, the accumulation of defects breaks charge neutrality and creates an electric field which is screened over the Debye length by depletion or accumulation of mobile charges..$^{21}$ The Debye length is thus the scale for charge inhomogeneities, over which changes in the density of mobile carriers can be expected, and determines the extension of the space-charge region at an interface or grain boundary. It has been already shown in different materials how their electrical resistances or electrochemical properties can be altered in this way by decreasing the system size to the nanometer range. ${ }^{22}$

The occurrence of space-charge effects in YSZ, and the possibility of enhancing its oxygen ion conductivity in nanometer sized YSZ grains or thin films, has been a subject of controversy in recent years. ${ }^{23-25}$ While some authors have reported increases in the ionic conductivity in samples with nanometer size grains, ${ }^{26}$ others have found no changes ${ }^{27,28}$ or even decreases ${ }^{29}$ of the conductivity in samples produced with different synthesis routes and with different grain sizes and dopant concentrations. ${ }^{18} \mathrm{O}$ tracer diffusion experiments assisted by secondary-ion mass spectroscopy also yield contradictory results while three orders of magnitude increase in the diffusion coefficient as compared with bulk samples has been reported in nanocrystalline thin films, ${ }^{30}$ bulk nanocrys- 
talline ceramics show no change. ${ }^{31}$ Moreover, the interpretations of the increases in conductivity in terms of spacecharge effect have to reconcile the fact that very small values of the Debye screening length (on the order of only $0.1 \mathrm{~nm}$ ) result for typical dopant concentrations $(8-10 \mathrm{~mol} \% \mathrm{YSZ})$ at intermediate temperatures $\left(500{ }^{\circ} \mathrm{C}\right)$.

Here we present a study of the effects of decreasing the grain size down to $17 \mathrm{~nm}$ on the oxide-ion conductivity of nanocrystalline YSZ ceramics. By using impedance spectroscopy we measured the frequency and temperature dependence of the complex impedance of samples with grain size ranging between 900 and $17 \mathrm{~nm}$ prepared by ball milling. As compared to previous studies of size effects in YSZ, this technique offers an efficient way of controlling grain size down to the nanometer range. ${ }^{32,33}$ While the specific grainboundary conductivity is rather independent of the grain size, we find a decrease in the bulk conductivity values when decreasing grain size. Our results show the existence of a space-charge region inside the grains, close to the grain boundary, that, contrary to what expected, extends several nanometers toward the grain core, and is responsible for a large depletion of mobile oxygen vacancies and therefore for a large decrease in the bulk oxide-ion conductivity in nanocrystalline YSZ.

\section{EXPERIMENTAL}

Nanocrystalline $10 \%$ mol yttria-stabilized zirconia samples, $\left(\mathrm{Y}_{2} \mathrm{O}_{3}\right)_{0.1}\left(\mathrm{ZrO}_{2}\right)_{0.9}$, with controlled grain size were obtained by mechanical milling a stoichiometric mixture of $\mathrm{ZrO}_{2}(99.9 \%$ purity, particle size $<40 \mu \mathrm{m})$ and $\mathrm{Y}_{2} \mathrm{O}_{3}$ (99.9\% purity, particle size $<40 \mu \mathrm{m}$ ), both commercial powders purchased from Alfa Aesar. The parameters of the milling process were the same used in other previously reported works: ${ }^{34}$ ball to powder ratio $7: 1$, rotation speed 250 rpm, and milling time $48 \mathrm{~h}$. In order to monitor the alloying process, $\mathrm{x}$-ray diffraction (XRD) patterns were recorded for different milling times, using a Xpert (Philips) diffractometer. The yttria content of the final powder was confirmed by $\mathrm{x}$-ray energy dispersive spectroscopy experiments using an energy dispersive x-ray analysis detector attached to a Philips XL-30 electron microscope. Powders obtained after milling for $48 \mathrm{~h}$ were uniaxially cold pressed into pellets (10 $\mathrm{mm}$ diameter and $1.5 \mathrm{~mm}$ thick), using $1 \mathrm{GPa}$ applied pressure. The density, determined by the Archimedes method, and the grain size of the samples were controlled using a sintering process known as two-step method. ${ }^{35}$ In this process the sample is first heated to a temperature $T_{1}$, then cooled down and held at a lower temperature $T_{2}$. The main advantage of this method is that grain growth is suppressed during the second stage, whereas significant densification takes place during both steps. For our samples, $T_{1}$ was chosen between 950 and $1150{ }^{\circ} \mathrm{C}$ while $T_{2}$ was $200{ }^{\circ} \mathrm{C}$ lower. The grain size of as-milled (green) samples was just $17 \mathrm{~nm}$ and the post sintering process allows increasing the grain size up to $100 \mathrm{~nm}$. The density also increases from $84 \%$ to about $93 \%$ of theoretical values after sintering. We find that the high pressure used and the two-step sintering process allows obtaining high-density values keeping the grain size in the nanometers range. An extensive x-ray diffraction line profile analysis of our samples and more experimental details can be found elsewhere. ${ }^{34}$ Atomic force microscopy (AFM) studies were performed with either a Nanoscope IIIa (Veeco) or a Pico 5100 (Agilent) equipment operating in tapping mode for selected samples in order to obtain complementary information about their surface morphology and microstructure. Also, a microcrystalline 10\% YSZ sample was prepared from the same as-milled powders by cold compaction at 1 $\mathrm{GPa}$, followed by a conventional sintering process at $1350{ }^{\circ} \mathrm{C}$, in air, during $20 \mathrm{~h}$.

$\mathrm{X}$-ray absorption measurements were performed at the BM25A SpLine beamline of the European Synchrotron Radiation Facility (ESRF). The storage ring was operated in the 16 bunch mode with a typical current of $90 \mathrm{~mA}$ at an electron-beam energy of $6 \mathrm{GeV}$. Measurements were performed at room temperature in the fluorescence detection mode on homogeneous thin layers of the powdered samples at both $\mathrm{Zr}$ and $\mathrm{Y} K$ edge. The monochromator used was a pseudochannel-cut type with two fixed Si (111) crystals in the $(-n,+n)$ configuration. Harmonic rejection was achieved by detuning the second crystal from the parallel alignment. The absorption spectra have been analyzed according to standard procedures ${ }^{36}$ by using the ATHENA which is included in the IFEFFIT program package for X-ray absorption spectroscopy (XAS) analysis. ${ }^{37}$

Complex conductivity measurements were carried out by using a HP $4284 \mathrm{LCR}$ analyzer in the $20 \mathrm{~Hz}-1 \mathrm{MHz}$ frequency range, with an applied ac voltage of $0.5 \mathrm{~V}$. Electrodes were deposited on pellet's flat surfaces by applying platinum paste. Stable electrical contacts were achieved by attaching platinum wires on top of the electrodes. For all the samples studied in this work, the electrical measurements were performed after heating the sample at $500{ }^{\circ} \mathrm{C}$ in air for $1 \mathrm{~h}$ to exclude any conductivity arising from water or other substances that might be absorbed during the synthesis process. Experimental conductivity and permittivity data were then collected at several fixed temperatures while cooling down the sample to room temperature. Electrical measurements were performed under air and under nitrogen atmosphere, yielding the same results in both cases.

\section{RESULTS AND DISCUSSION}

We present in this work electrical conductivity measurements of $10 \%$ mol-YSZ samples with four different grain sizes between 17 and $900 \mathrm{~nm}$. In a previous work, grain-size values of these samples were determined from a WarrenAverbach line profile analysis of the XRD patterns. ${ }^{34}$ Complementary AFM experiments were also performed in order to obtain an independent estimate of the grain size and compare it with the values obtained from XRD. We found in all cases a good agreement between both techniques (see Fig. 1). The values for the nominal average size of the grains as obtained from the XRD data are summarized in Table I, together with the temperatures used in the two-step sintering process detailed in the experimental section. The lattice parameter obtained from XRD data was found to be $5.146 \pm 0.003 \AA$ for all the samples, independent of the 

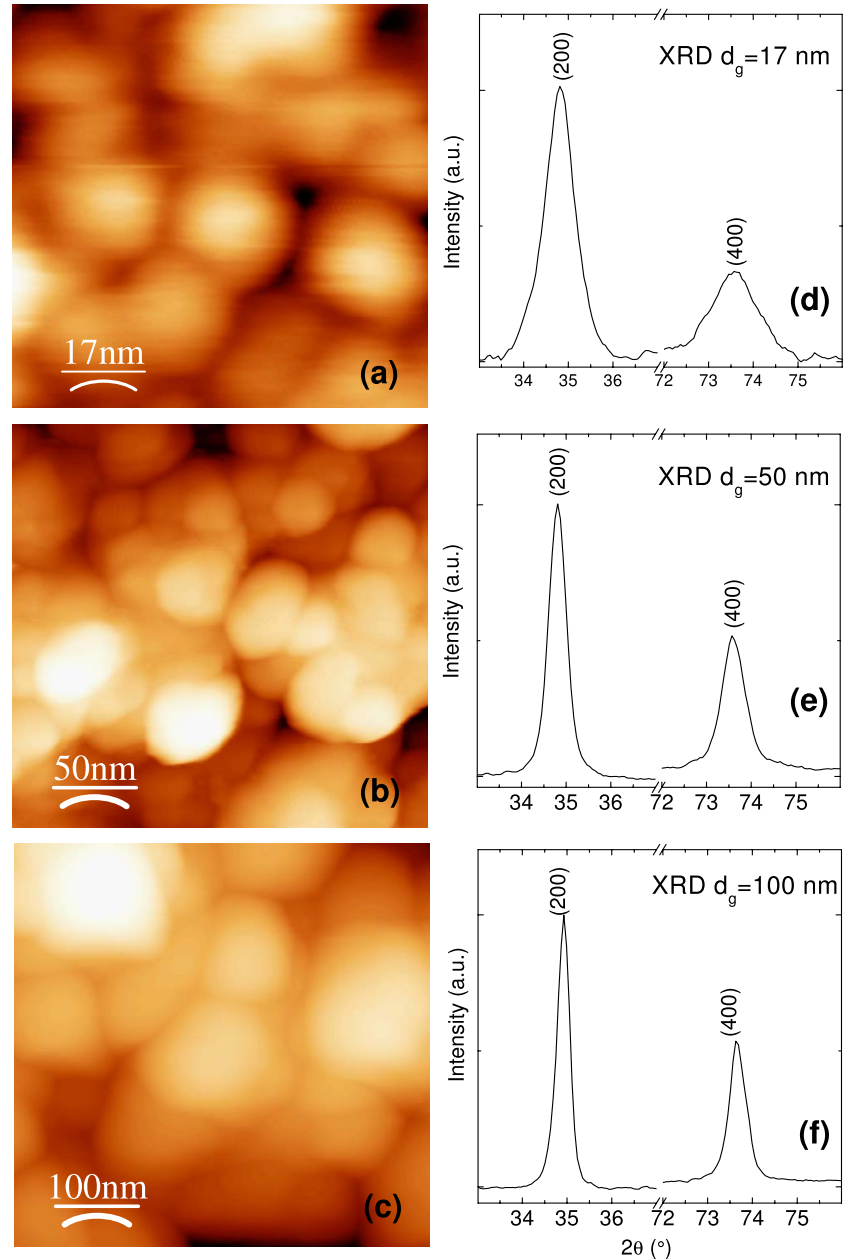

FIG. 1. (Color online) Atomic force microscope images for nanostructured samples with nominal grain size of (a) $17 \mathrm{~nm}$, (b) 50 $\mathrm{nm}$, and (c) $100 \mathrm{~nm}$. Corresponding x-ray diffraction peaks are shown in (d)-(f), showing broader peaks as grain size decreases.

grain size, and in agreement with that previously reported for YSZ.

In the following we present the results of electrical measurements on our samples. Complex conductivity as a function of frequency, $\sigma^{*}(\omega)=\sigma^{\prime}(\omega)+j \omega \varepsilon_{0} \varepsilon^{\prime}(\omega)$, was directly obtained from the measurements, with $\sigma^{\prime}$ its real part, $\varepsilon_{0}$ the permittivity of vacuum, and $\varepsilon^{\prime}$ the real part of the complex dielectric permittivity $\varepsilon^{*}(\omega)=\sigma^{*}(\omega) / j \omega \varepsilon_{0}$. Figure 2 shows the frequency dependence of (a) the real part of the conductivity $\sigma^{\prime}$ and (b) the real part of the permittivity $\varepsilon^{\prime}$, for the 50 $\mathrm{nm}$ grain-size sample, at several fixed temperatures. The data

TABLE I. Grain sizes, densities, and sintering temperatures of the samples studied in this work.

\begin{tabular}{crrc}
\hline \hline Grain size $(\mathrm{nm})$ & $T_{1}\left({ }^{\circ} \mathrm{C}\right)$ & $T_{2}\left({ }^{\circ} \mathrm{C}\right)$ & Density $(\%$ theoretical $)$ \\
\hline $17 \pm 2$ & & & $84.0 \pm 0.5$ \\
$50 \pm 5$ & 1050 & 850 & $91.2 \pm 0.2$ \\
$100 \pm 10$ & 1150 & 950 & $92.7 \pm 0.2$ \\
$900 \pm 100$ & 1350 & 1350 & $93.0 \pm 0.2$ \\
\hline \hline
\end{tabular}
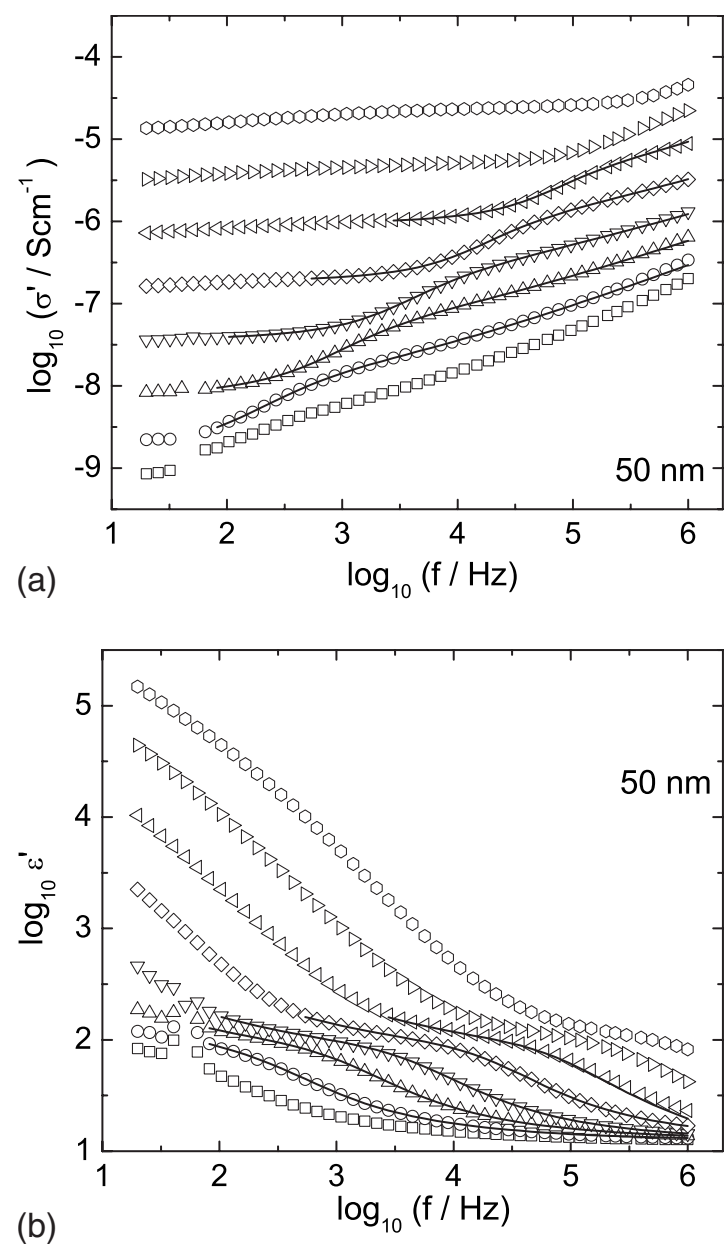

FIG. 2. (a) Real part of conductivity $\left(\sigma^{\prime}\right)$ versus frequency of the $50 \mathrm{~nm}$ grain-size sample. Isothermal data sets were measured at $477,505,535,570,610,655,709$, and $772 \mathrm{~K}$ from bottom to top. (b) Real part of permittivity $\left(\varepsilon^{\prime}\right)$ versus frequency for the same sample [at the same temperatures shown in (a)].

clearly show the presence of the bulk and grain-boundary contributions to the conductivity and permittivity characteristic of polycrystalline ionic conductors. ${ }^{38,39}$ While the bulk contribution is dominant at high frequencies and/or low temperatures, the grain-boundary contribution dominates at low frequencies or high temperatures. At the lowest frequencies and highest temperatures the effect of electrode polarization is also visible giving rise to a large increase in the apparent permittivity and a decrease in the measured conductivity. As expected, due to small size of the grains in this sample, the frequency range where the bulk response is dominant is more limited than in microcrystalline samples. This is because in the nanocrystalline samples the mobile ions reach more easily the grain boundaries, getting blocked at shorter times (i.e., higher frequencies). In fact, while an almost frequencyindependent conductivity (the so-called conductivity plateau) is observed at the frequencies where the grain-boundary contribution dominates, the characteristic plateau of the bulk conductivity is hardly visible in the $\sigma^{\prime}$ vs $f$ plots since the conductivity starts to decrease due to the blocking of ions at the grain boundaries. This effect is even more pronounced for the sample with smallest grain size, $17 \mathrm{~nm}$, as shown in 

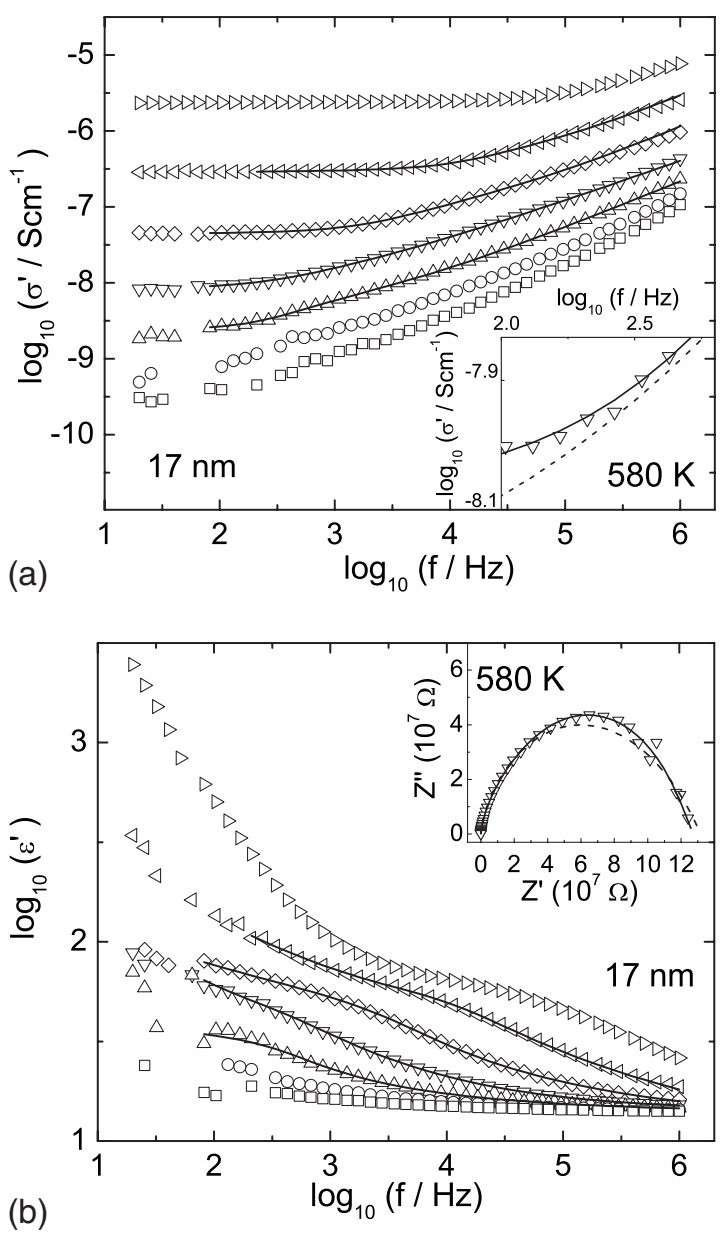

FIG. 3. (a) Real part of conductivity versus frequency of the 17 $\mathrm{nm}$ grain-size sample. Isothermal data sets were measured at 467 , $500,537,580,630,690$, and $769 \mathrm{~K}$ from bottom to top. (b) Real part of permittivity versus frequency for the same sample [at the same temperatures shown in (a)]. Insets show results of fitting data to expression (2); with two contributions (solid line) and with one contribution (discontinuous line) (see text).

Fig. 3, where (a) conductivity and (b) permittivity data are plotted as a function of frequency. Thus, in order to obtain the dc bulk $\left(\sigma_{b}\right)$ as well as the grain-boundary $\left(\sigma_{g b}\right)$ conductivity of the samples at each temperature, we fitted the data by using a complex nonlinear least-squares method to an equivalent circuit where both contributions are in series,

$$
\frac{1}{\sigma^{*}}=\frac{1}{\sigma_{b}^{*}}+\frac{1}{\sigma_{g b}^{*}},
$$

where each contribution ( $i=b$ and $g b$, respectively, for bulk and grain-boundary contribution) is modeled by using the following complex conductivity function: ${ }^{40}$

$$
\sigma_{i}^{*}=\sigma_{i}+Q_{i}(j \omega)^{n_{i}}+j \omega \varepsilon_{0} \varepsilon_{i} .
$$

This function contains a power-law frequency-dependent term $Q_{i}$ (universal Jonscher's response) as usually found in ionic conductors ${ }^{41}$ to account for the dispersive conductivity observed at frequencies higher than that where the characteristic plateau is observed [note the linear dependence with slope $n$ in the double-logarithmic plots of Figs. 2(a) and 3(a) arising from such a power-law behavior]. There was no need to include an additional term in Eq. (2) to account for a linear frequency-dependent conductivity, the so called nearly constant loss regime characteristic of ionically conducting materials at very low temperature or high frequencies ${ }^{42,43}$ since it was not present in the temperature and frequency ranges analyzed in this work. The result from these fits to Eqs. (1) and (2) are also shown in Figs. 2 and 3, showing an excellent agreement with experimental data. We want to remark that, even for the $17 \mathrm{~nm}$ grain-size sample, where the bulk contribution is strongly hidden by the grain-boundary contribution due to the small grain size, the fits clearly show that both contributions are present in the experimental data. This is better observed in the inset of Fig. 3(b), where the plot of the imaginary part of the impedance as a function of the real part shows that experimental data cannot be described by assuming just a bulk contribution. It can be also observed that the fits allow obtaining both the bulk and grain-boundary conductivity values even for this sample with the smallest grain size. This allowed us to obtain the temperature dependence of the bulk dc conductivity $\left(\sigma_{b}\right)$ and grain-boundary conductivity $\left(\sigma_{g b}\right)$ for samples with different grain sizes.

Results of the temperature dependence of the bulk conductivity $\sigma_{b}$ are shown as Arrhenius plots in Fig. 4(a). For comparison, we have also plotted in the same graph the conductivity values corresponding to a $9.5 \%$ mol-YSZ singlecrystalline sample. ${ }^{44,45}$ A thermally activated behavior of the ionic conductivity is clearly observed for all the samples, showing similar values of the activation energy $E_{b}$ for the different grain sizes (see Table II). Interestingly, the sample with the smallest grain size shows the lowest value of $E_{b}$. On the other hand, a large decrease of about two orders of magnitude is found for $\sigma_{b}$, at a given temperature, when the grain size decreases from 900 to $17 \mathrm{~nm}$. Moreover, such a decrease is steeper as the size decreases toward the nanometer range. Similar activation energies $E_{g b}$ are also found for the grainboundary conductivity $\left(\sigma_{g b}\right)$ for the different samples, independent of the grain size [see Table II and inset of Fig. 4(b)]. In order to compare the possible influence of the grain size on the conductivity at the grain boundary, it is necessary to correct for the intrinsic (geometrical) dependence of the measured grain-boundary conductivity $\sigma_{g b}$ on grain size. ${ }^{46}$ Using the brick layer model approximation, ${ }^{46,47}$ we have estimated the specific grain-boundary conductivity $\sigma_{s g b}$, from our measurements. This model assumes that the permittivity value at the grain boundary is similar to the bulk one, $\varepsilon_{g b}=\varepsilon_{b}$. One can thus estimate the specific grain-boundary conductivity as $\sigma_{s g b}=\sigma_{g b}\left(C_{b} / C_{g b}\right)$, being $\left(C_{b} / C_{g b}\right)$ the ratio between the measured bulk and grain-boundary capacitance values. Despite the approximations made in getting these estimates, it is quite remarkable that we found the specific grain-boundary conductivity $\sigma_{s g b}$ does not depend on the grain size of the samples [see main panel of Fig. 4(b)]. Therefore, our results show that oxide-ion dynamics at the grain boundaries is similar in all samples, yielding the same specific grainboundary conductivity, while bulk conductivity decreases significantly as a result of decreasing the grain size below $100 \mathrm{~nm}$ to the nanometer range.

It is also remarkable that the activation energies for bulk dc conductivity $\left(E_{b}\right)$ and grain-boundary conductivity $\left(E_{g b}\right)$ 

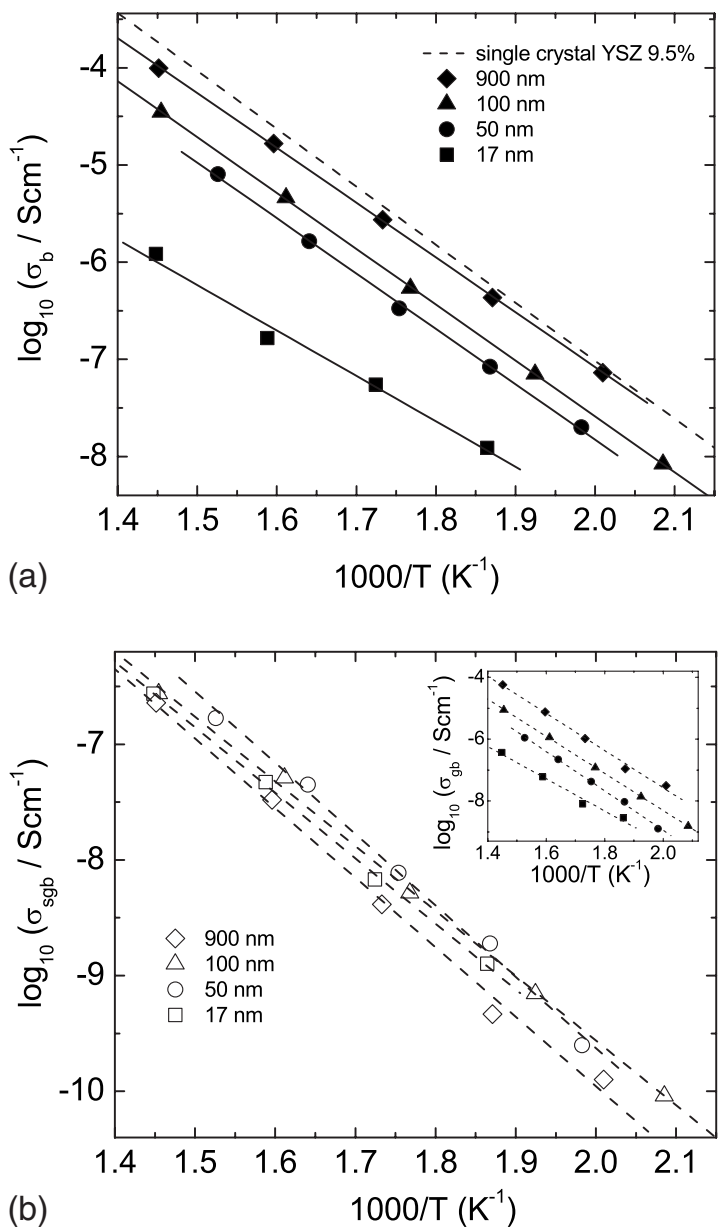

FIG. 4. (a) Bulk ionic dc conductivity versus inverse temperature for samples with different grain sizes: $17 \mathrm{~nm}, \boldsymbol{0} 50 \mathrm{~nm}, \boldsymbol{\Delta}$ $100 \mathrm{~nm}$, and $\$ 900 \mathrm{~nm}$. The conductivity data of a $9.5 \%$ mol-YSZ single-crystalline sample (Ref. 39) have been included for comparison. (b) Specific grain boundary (main panel) and grain-boundary (inset) conductivities versus inverse temperature for the same set of samples.

are found to be very similar. This points to the absence of different phases or contaminants in the grain-boundary region..$^{46,48}$ Therefore, the ion blocking observed at the grain boundary would not be due to any extrinsic effect but just to the existence of a space-charge layer (SCL) close to the grain boundary, where mobile ion concentration is depleted. Yttrium segregation to the grain boundary is known to occur in YSZ ceramics giving rise to enhanced oxygen vacancy concentrations at the grain-boundary core ${ }^{46}$ and blocking charge

TABLE II. Bulk, grain boundary, and specific grain-boundary activation energies for the samples studied in this work.

\begin{tabular}{cccc}
\hline \hline Sample $(\mathrm{nm})$ & $E_{b}(\mathrm{eV})$ & $E_{g b}(\mathrm{eV})$ & $E_{s g b}(\mathrm{eV})$ \\
\hline 17 & $0.93 \pm 0.04$ & $1.03 \pm 0.02$ & $1.12 \pm 0.02$ \\
50 & $1.13 \pm 0.02$ & $1.26 \pm 0.02$ & $1.20 \pm 0.02$ \\
100 & $1.13 \pm 0.02$ & $1.18 \pm 0.02$ & $1.10 \pm 0.02$ \\
900 & $1.12 \pm 0.02$ & $1.19 \pm 0.02$ & $1.19 \pm 0.02$ \\
\hline \hline
\end{tabular}

TABLE III. $\Delta \varphi(0)=\varphi(0)-\varphi(\infty)$ potential of the grain-boundary core relative to the bulk; $\lambda^{*}$ is the space-charge layer thickness, and $L_{D}$ is the Debye length estimated from Eq. (4) at $575 \pm 5 \mathrm{~K}$ for each sample.

\begin{tabular}{cccc}
\hline \hline Sample $(\mathrm{nm})$ & $\Delta \varphi(0)(\mathrm{V})$ & $\lambda^{*}(\mathrm{~nm})$ & $L_{D}(\mathrm{~nm})$ \\
\hline 17 & 0.11 & 7.20 & 2.43 \\
50 & 0.16 & 3.75 & 1.05 \\
100 & 0.18 & 2.43 & 0.65 \\
900 & 0.23 & 1.78 & 0.40 \\
\hline \hline
\end{tabular}

flow. In fact, oxygen vacancies accumulate in an amount higher than that expected from stoichiometry, yielding a positive charge there and a consequent depletion of the grain-boundary region (inside the grains but close to the grain surface) from mobile oxygen vacancies, thus forming a SCL. This SCL which in YSZ microcrystalline ceramics has been proposed to be on the order of a few nanometers ${ }^{46-49}$ might provide an explanation for the observed decrease in bulk conductivity in nanometer sized grains. For small grains the extension of the SCL might be large enough to make the volume of the SCL region be comparable to the volume of the rest of the grain. This would explain an effective decrease in the mobile ion concentration in the bulk of the grain and a corresponding decrease in its conductivity. In particular, in the case of the sample with a grain size of $17 \mathrm{~nm}$, a SCL only $1.7 \mathrm{~nm}$ thick would occupy a similar volume as the rest of the grain, leading to an approximate halving of the mobile oxygen vacancy concentration in the bulk, with the remaining half segregated to the grain-boundary core. If the SCL thickness is even larger, the decrease in oxide-ion conductivity can be quite significant, as we indeed have observed in our samples.

The brick layer model approximates the thickness of the SCL in polycrystalline ceramics to half the width of the grain-boundary region since it is shared by the two neighboring grains at the boundary. ${ }^{46}$ Assuming that the ceramic grains in the material are cubes and that the average grainboundary thickness $\delta_{g b}$ can be estimated in terms of the ratio of bulk and grain-boundary capacitances, $\delta_{g b}=C_{b} / C_{g b} d_{g}$, with $d_{g}$ the average size of the grains, the thickness of the space-charge layer $\lambda^{*}$ is estimated as $\lambda^{*} \approx \delta_{g b} / 2$. The values obtained for the samples studied in this work are shown in Table III, and found to be in the range 1.78 and $7.2 \mathrm{~nm}$ when grain size decreases from 900 to $17 \mathrm{~nm}$. The increase in SCL thickness as the grain size decreases might just reflect the fact that the charge segregated to the grain-boundary core coincides with the amount of charge depleted from the SCL volume. In fact, if we calculate the concentration of charge per unit area at the grain-boundary core for the different samples as the charge depleted from the SCL (nominal oxygen vacancy concentration in the bulk, $5.3 \mathrm{~nm}^{-3}$, times the SCL volume) divided by the grain-boundary area (assuming spherical grains), we obtain a similar value of $13.5 \pm 3 \mathrm{~nm}^{-2}$ for all the samples, although the total area of the grain boundary is changing by a factor higher than $10^{3}$ when the grain size is decreased from 900 to $17 \mathrm{~nm}$. This finding is consistent with the idea that segregation of yttrium and va- 

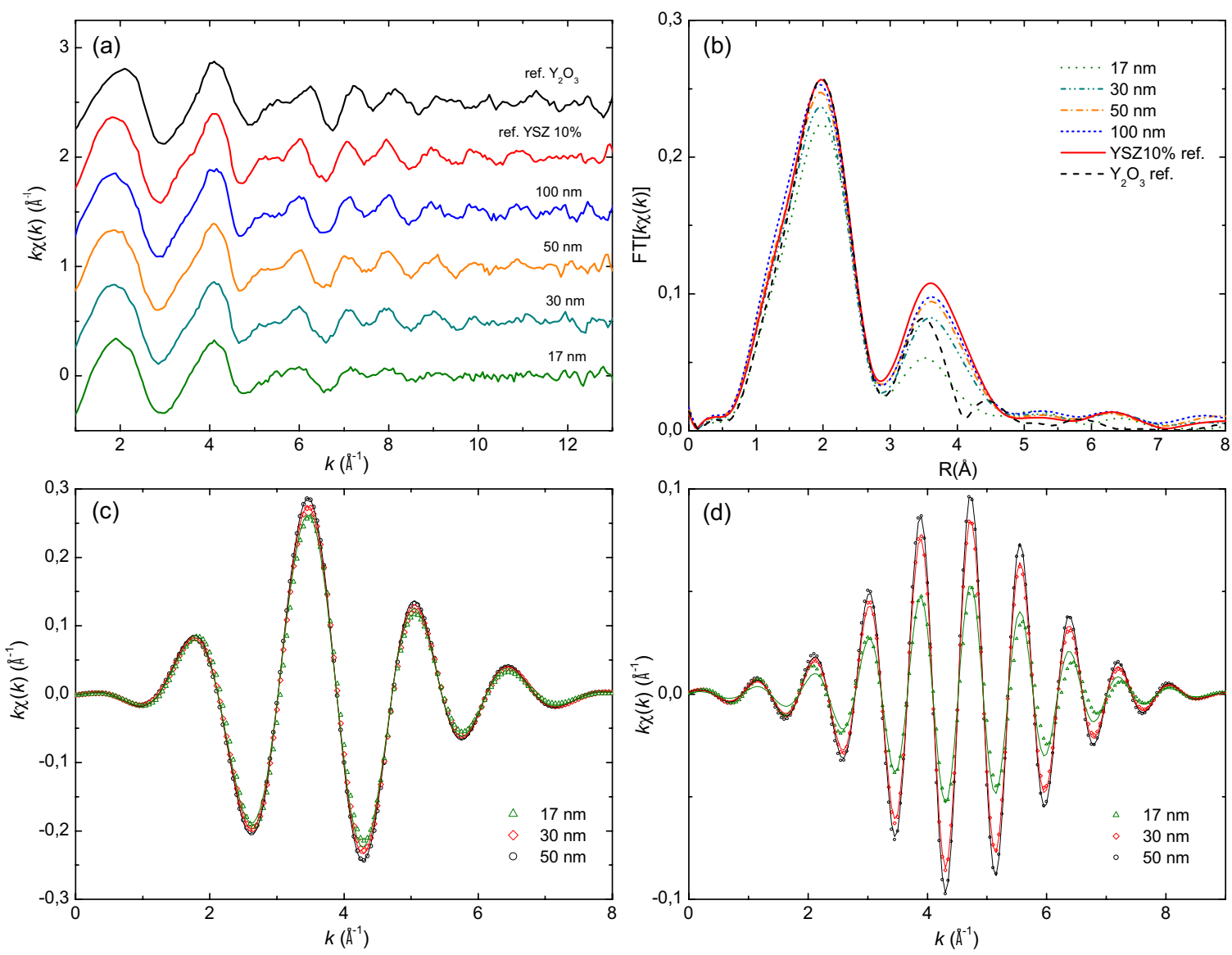

FIG. 5. (Color online) (a) Comparison of the Y $K$-edge $k \chi(k)$ EXAFS spectra of the YSZ nanocrystalline samples with grain sizes ranging from 100 to $17 \mathrm{~nm}$. The EXAFS signals of reference compounds $\mathrm{Y}_{2} \mathrm{O}_{3}$ and $10 \% \mathrm{~mol} \mathrm{YSZ}$ are also included. The spectra have been vertically shifted for the sake of clarity but preserving their relative scales. (b) Comparison of the modulus of the Fourier transform of the experimental $k \chi(k)$ EXAFS spectra shown in the previous panel. (c) Fourier filtered $k \chi(k)$ signals of the first coordination shell in the case of the $50 \mathrm{~nm}(\bigcirc), 30 \mathrm{~nm}(\diamond)$, and $17 \mathrm{~nm}(\triangle)$ samples. The Fourier filtered contribution of the second coordination shell is shown in panel (d). In both (c) and (d), the solid lines correspond to the same contribution in the $100 \mathrm{~nm}$ sample after applying an amplitude reduction factor (see text for details).

cancies is essentially determined by thermodynamics and kinetics and should not depend much on the size of the grains. In these estimates we considered the SCL is fully depleted of mobile oxygen vacancies. While this may not be exactly true, it may be regarded to be a good approximation provided vacancy concentration decreases continuously from the nominal bulk value at the center of the grain toward a value several orders of magnitude lower at the grain boundary. ${ }^{46}$

In order to investigate if indeed the microstructure and composition of the grain boundary is similar for grains of different sizes, as expected from the analysis of the electrical conductivity measurements, we have performed a detailed XAS study. Figure 5 shows the comparison of the Y $K$ edge $k$-weighted extended $\mathrm{x}$-ray-absorption fine structure (EXAFS) signals and their Fourier transforms (FTs) for samples with different grain sizes. In all the cases the EXAFS signal, $\chi(k)$, was obtained after removing the background by a cubic spline polynomial fitting and by normalizing the magnitude of the oscillations to the edge jump. The corresponding pseudoradial distribution function around the absorbing atom has been obtained by performing the Fourier transform (using a sine window) of the $k \chi(k)$ EXAFS signals. For a compara- tive study, the Fourier transform of EXAFS signal was performed within the same $k$ range. The obtained signals are consistent with those previously reported.$^{50,51}$ As shown in Fig. 5(a), the EXAFS signals are similar for all the samples, showing the same spectral features (perfect coincidence into the energy position, relative intensities, and shape), and the only remarkable difference among them is a progressive damping of the amplitude as the grain size decreases. This is made clear by comparing the Fourier transforms in Fig. 5(b). Again, one finds the same shape but the intensity of the main peaks changes with the grain size. It is important to note the fact that the reduction in the FT intensity is quite different for the first and the second peaks. This can be better observed in the comparison of the back Fourier transforms performed after Fourier filtering these contributions in the range 0.54 $<R<2.87$ and $2.87<R<4.73$. As shown in panel (c) of Fig. 5, the contribution of the first coordination shell shows the same frequency, i.e., same interatomic distance, in all the samples and only its amplitude decreases upon reducing the grain size. Taking as a reference the $100 \mathrm{~nm}$ sample, this amplitude is about $98 \%, 94 \%$, and $90 \%$ in the case of the 50 , 30 , and $17 \mathrm{~nm}$ samples, respectively. Indeed, as shown in the 
figure, the filtered signals of these compounds can be perfectly reproduced by applying this reduction amplitude factor to that of the $100 \mathrm{~nm}$ sample. By contrast, the amplitude of the second peak contribution is reduced to $\sim 95 \%, 87 \%$, and $55 \%$ for the 50,30 , and $17 \mathrm{~nm}$ samples, respectively [see panel (d) in Fig. 5]. The first FT peak corresponds to the next neighboring $\mathrm{Zr}(\mathrm{Y})-\mathrm{O}$ coordination while the second is related to the second coordination sphere, $\mathrm{Zr}(\mathrm{Y})-\mathrm{Zr}(\mathrm{Y})$. Therefore, this behavior indicates that the local structure around the absorbing Y has a similar oxygen arrangement (similar results, not shown, have been found in the case of the $\mathrm{Zr} \mathrm{K}$ edge). The fact that no significant difference is found even for the EXAFS of the sample with $17 \mathrm{~nm}$ grain size, in which the boundary region has the largest contribution, indicates that the local order is similar in both bulk and grainboundary regions. The decrease in the FT intensity is progressive and scales with the grain size, which suggests that the disorder in the grain boundary slightly increases with respect to that of the bulk. The fact that the differences are greater in the intensity of the second FT peak is consistent with this view. At the grain boundary, the coordination of the absorbing atoms decreases due to the surface effect without affecting the local structure. Consequently, as the presence of these boundaries becomes more important into the EXAFS, i.e., when the size decreases, the effect is enhanced. Most important is that XAS measurements show that the microstructure and composition of the grain boundary are similar for the different samples (even for that with the smallest grain size) and no departure of its electrical properties is expected, in agreement with our finding of a similar specific grain-boundary conductivity.

Finally, it is also possible to estimate the Debye length $L_{D}$ (or screening length) from the values obtained for the SCL thickness $\lambda^{*}$. The Debye length is defined as usual ${ }^{46}$ by

$$
L_{D}=\sqrt{\frac{\varepsilon_{r} \varepsilon_{0} k_{B} T}{Z e^{2} n}}
$$

with $e$ the charge of the electron, $Z=2$ for oxide ions, $k_{B}$ the Boltzmann constant, and $n$ the concentration of mobile oxygen vacancies. Assuming a constant acceptor concentration up to the grain-boundary core (Mott-Schottky approximation) and solving the Poisson equation, the following expression is obtained:

$$
L_{D}=\frac{\lambda^{*}}{2} \sqrt{\frac{k_{B} T}{e \Delta \varphi(0)}},
$$

where $\Delta \varphi(0)$ is the grain-boundary space-charge potential calculated from the ratio between $\sigma_{b}$ and $\sigma_{s g b} .{ }^{46}$ The Debye length, estimated by using expression (4) and the parameters obtained from electrical conductivity measurements, takes values between 0.4 and $2.43 \mathrm{~nm}$ in all our nanocrystalline samples (see Table III), much larger than the value on the order of $0.1 \mathrm{~nm}$ expected for $10 \%$ mol-YSZ according to Eq. (3) with the corresponding bulk concentration of oxygen vacancies. Similar discrepancies, of about a factor of 4 , between the values obtained for the Debye length by using either Eq. (3) or Eq. (4) have been obtained in other systems $^{46}$ and have been ascribed to the approximations in-

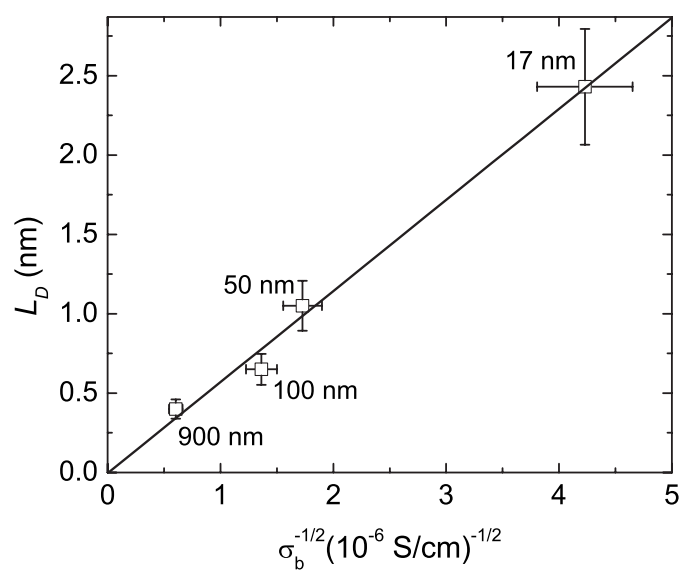

FIG. 6. Estimated Debye length for each sample vs the inverse square root of measured bulk ionic dc conductivity. Solid line is a linear fit (see text).

volved in the brick layer model that is used to estimate the corresponding parameters in Eq. (4). However, this cannot easily explain the much larger increase in the Debye length when decreasing the grain size.

Interestingly, the large values obtained for the Debye length in the samples with smallest grain size can be rationalized in terms of an effective decrease in the concentration of oxygen vacancies, as pointed out above, as the volume of $\mathrm{SCL}$ relative to the total grain increases. That is, in the sample with smallest grain size, $17 \mathrm{~nm}$, the $7 \mathrm{~nm}$ thick SCL amounts about $99 \%$ of the total grain volume and as a consequence, the grain is strongly depleted from oxygen vacancies. Since the bulk conductivity $\sigma_{b}$ is proportional to the concentration of oxygen vacancies, this would result in a drastic decrease in the conductivity of the grain compared to values of single-crystal or microcrystalline ceramic samples. Actually this is observed in our samples as illustrated by the decrease in conductivity with grain size depicted in Fig. 4(a). Interestingly, according to Eq. (3), a linear dependence observed in plots of the Debye length as a function of $\sigma_{b}^{-1 / 2}$ would suggest that, beyond the carrier depletion in the spacecharge region, an overall decrease in the carrier density may occur in samples with small grain size. In these samples, where the space charge is a significant fraction of the grain volume, the reduced (measured) value of $\sigma_{b}$ may be reflecting an effective value of the charge density which is well below the nominal values expected from stoichiometry. Figure 6 shows that indeed this linear relationship holds nicely for the samples in the whole range of grain sizes analyzed, from 900 to $17 \mathrm{~nm}$.

\section{SUMMARY AND CONCLUSIONS}

We have prepared and electrically characterized nanocrystalline $10 \%$ mol yttria-stabilized zirconia samples with varying grain sizes from $900 \mathrm{~nm}$ down to $17 \mathrm{~nm}$. By using ball milling and a subsequent two-step sintering process we were able to accurately control the grain size down to the nanometer range while attaining high-density values at the same time. By using impedance spectroscopy we measured the 
oxide-ion conductivity of the different samples and were able to separate and determine the bulk and grain-boundary conductivities as a function of grain size. We found that the specific grain-boundary conductivity, i.e., the conductivity associated to oxide ions traveling through the grain-boundary core from one grain to another one is similar for all samples and thus rather independent of the grain size. However, the bulk conductivity related to oxide ions diffusing inside each grain decreases strongly as the grain size is decreased below $100 \mathrm{~nm}$ in diameter. Both findings are shown to be consistent with the formation of a space-charge layer inside the grains, close to the grain boundaries, whose thickness increases rapidly when the grain size is reduced to the nanometer range. The similar specific grain-boundary conductivities observed experimentally indicate similar composition (segregated charge density) for the grain-boundary core in all samples as dictated by thermodynamics and kinetics, and confirmed also by EXAFS measurements. On the other hand, in order the segregated charge density to be similar, it is necessary that the space-charge layer thickness increases as the grain surface reduces with its size. The decrease observed in the measured bulk conductivity by two orders of magnitude when decreasing the grain size from 900 to $17 \mathrm{~nm}$ points to an ensuing decrease in the oxygen vacancy concentration inside the grains.

\section{ACKNOWLEDGMENTS}

We acknowledge financial support by Junta de Comunidades de Castilla-La Mancha through Project No. PAI-05013, by CAM under Grant No. S2009/MAT-1756 (Phama), by Spanish MICINN through Grants No. MAT2008-06517C02, No. MAT2008-06542-C04, and No. FIS2009-12964C05-04, and Consolider Ingenio 2010 under Grant No. CSD2009-00013 (Imagine). We thank J. Garcia-Barriocanal for helpful discussions. We are indebted to the staff of SpLine for their help during the experimental work at ESRF.
${ }^{1}$ A. V. Chadwick, Nature (London) 408, 925 (2000).

${ }^{2}$ B. C. H. Steele and A. Heinzel, Nature (London) 414, 345 (2001).

${ }^{3}$ P. Lacorre, F. Goutenoire, O. Bohnke, R. Retoux, and Y. Laligant, Nature (London) 404, 856 (2000).

${ }^{4}$ J. B. Goodenough, Nature (London) 404, 821 (2000).

${ }^{5}$ T. H. Etsell and S. N. Flengas, Chem. Rev. 70, 339 (1970).

${ }^{6}$ N. P. Brandon, S. Skinner, and B. C. H. Steele, Annu. Rev. Mater. Res. 33, 183 (2003).

${ }^{7}$ Z. Shao and S. M. Haile, Nature (London) 431, 170 (2004).

${ }^{8}$ Y.-H. Huang, R. I. Das, Z.-L. Xing, and J. B. Goodenough, Science 312, 254 (2006).

${ }^{9}$ S. Tao and J. T. S. Irvine, Nat. Mater. 2, 320 (2003).

${ }^{10}$ B. A. Boukamp, Nat. Mater. 2, 294 (2003).

${ }^{11}$ G. Kim, Appl. Phys. Lett. 88, 024103 (2006).

${ }^{12}$ H. L. Tuller, Solid State Ionics 131, 143 (2000).

${ }^{13}$ J. Maier, Nat. Mater. 4, 805 (2005).

${ }^{14}$ J. Maier, Solid State Ionics 175, 7 (2004).

${ }^{15}$ F. Berkemeier, Mohammad Reza Shoar Abouzari, and G. Schmitz, Phys. Rev. B 76, 024205 (2007).

${ }^{16}$ L. Schlapbach and A. Zuttel, Nature (London) 414, 353 (2001).

${ }^{17}$ A. C. Dillon, K. M. Jones, T. A. Bekkedahl, C. H. Kiang, D. S. Betune, and M. J. Heben, Nature (London) 386, 377 (1997).

${ }^{18}$ S. R. Ramanathan, J. Vac. Sci. Technol. A 27, 1126 (2009).

${ }^{19}$ J. Maier, J. Electrochem. Soc. 134, 1524 (1987).

${ }^{20}$ J. Maier, Solid State Ionics 157, 327 (2003).

${ }^{21}$ K. L. Kliewer and J. S. Koehler, Phys. Rev. 140, A1226 (1965).

${ }^{22}$ J. Maier, Phys. Chem. Chem. Phys. 11, 3011 (2009).

${ }^{23}$ S. J. Litzelman, J. L. Hertz, W. Jung, and H. Tuller, Fuel Cells 8, 294 (2008)

${ }^{24}$ A. Karthikeyan, Ch. L. Chan, and L. Ramanathan, Appl. Phys. Lett. 89, 183116 (2006).

${ }^{25}$ J. Garcia-Barriocanal, A. Rivera-Calzada, M. Varela, Z. Sefrioui, E. Iborra, C. León, S. J. Pennycook, and J. Santamaría, Science 321, 676 (2008).

${ }^{26}$ I. Kosacki, T. Suzuki, V. Petrovsky, and H. U. Anderson, Solid
State Ionics 136-137, 1225 (2000).

${ }^{27}$ C. Peters, M. Bockmeyer, R. Krüger, A. Weber, and E. IversTiffee, in Current and Future Trends of Functional Oxide Films, edited by D. Kumar et al., Materials Research Society Symposium Proceedings Vol. 928 (Materials Research Society, Warrendale, Pennysylvania, 2006), pp. 1-7.

${ }^{28}$ P. Mondal, A. Klein, W. Jaegermann, and H. Hahn, Solid State Ionics 118, 331 (1999).

${ }^{29}$ S. S. Jiang, W. A. Schulze, V. R. W. Amarakoon, and G. C. Stangle, J. Mater. Res. 12, 2374 (1997).

${ }^{30}$ G. Knöner, K. Reimann, R. Roewer, U. Soedervall, and H.-E. Schaefer, Proc. Natl. Acad. Sci. U.S.A. 100, 3870 (2003).

${ }^{31}$ R. A. De Souza, M. J. Pietrovski, U. Anselmi-Tamburini, S. Kim, Z. A. Munir, and M. Martín, Phys. Chem. Chem. Phys. 10, 2067 (2008).

${ }^{32}$ N. J. Kidner, N. H. Perry, T. O. Mason, and E. J. Garboczi, J. Am. Ceram. Soc. 91, 1733 (2008).

${ }^{33}$ C. Peters, A. Weber, B. Butz, D. Gerthsen, and E. Ivers-Tiffée, J. Am. Ceram. Soc. 92, 2017 (2009).

${ }^{34}$ O. J. Durá and M. A. López de la Torre, J. Phys. D 41, 045408 (2008).

${ }^{35}$ I.-Wei Chen and X. H. Wang, Nature (London) 404, 168 (2000).

${ }^{36} X$-Ray Absorption: Principles, Applications, Techniques of EXAFS, SEXAFS, and XANES, edited by D. C. Koningsberger and R. Prins (Wiley-Interscience, New York, 1988).

${ }^{37}$ B. Ravel and M. Newville, J. Synchroton Radiat. 12, 537 (2005).

${ }^{38}$ A. Pimenov, J. Ullrich, P. Lunkenheimer, A. Loidl, and C. H. Ruscher, Solid State Ionics 109, 111 (1998).

${ }^{39}$ A. Rivera, J. Santamaría, and C. León, Appl. Phys. Lett. 78, 610 (2001).

${ }^{40}$ J. R. Macdonald, Impedance Spectroscopy. Theory, Experiments and Applications, 2nd ed. (Wiley-Interscience, Hoboken, New Jersey, 2005).

${ }^{41}$ A. K. Jonscher, Dielectric Relaxation in Solids (Chelsea Dielectrics, London, 1983). 
${ }^{42}$ C. León, A. Rivera, A. Várez, J. Sanz, J. Santamaria, and K. L. Ngai, Phys. Rev. Lett. 86, 1279 (2001).

${ }^{43}$ K. L. Ngai, J. Chem. Phys. 110, 10576 (1999).

${ }^{44}$ C. León, M. L. Lucía, and J. Santamaría, Phys. Rev. B 55, 882 (1997).

${ }^{45}$ C. León, M. L. Lucía, J. Santamaría, and F. Sánchez-Quesada, Phys. Rev. B 57, 41 (1998).

${ }^{46}$ X. Guo and R. Waser, Prog. Mater. Sci. 51, 151 (2006).

${ }^{47}$ T. van Dijk and A. J. Burggraaf, Phys. Status Solidi A 63, 229
(1981).

${ }^{48}$ X. Guo, W. Sigle, and J. Maier, J. Am. Ceram. Soc. 86, 77 (2003).

${ }^{49}$ A. Tschöpe, E. Sommer, and R. Birringer, Solid State Ionics 139, 255 (2001).

${ }^{50}$ P. Li, I.-W. Chen, and J. E. Penner-Hahn, Phys. Rev. B 48, 10063 (1993).

${ }^{51}$ M. Gateshki, V. Petkov, G. Williams, S. K. Pradhan, and Y. Ren, Phys. Rev. B 71, 224107 (2005). 